\title{
Biofilms of non-Candida albicans Candida species: quantification, structure and matrix composition
}

\author{
SÓNIA SILVA*, MARIANA HENRIQUES*, ANTÓNIO MARTINS*, ROSÁRIO OLIVEIRA*, DAVID WILLIAMS† \& \\ JOANA AZEREDO* \\ *Institute for Biotechnology and Bioengineering, Universidade do Minho, Braga, Portugal, and †School of Dentistry, Cardiff University, \\ Heath Park, Cardiff, UK
}

\begin{abstract}
Most cases of candidiasis have been attributed to C. albicans, but recently, non-Candida albicans Candida (NCAC) species have been identified as common pathogens. The ability of Candida species to form biofilms has important clinical repercussions due to their increased resistance to antifungal therapy and the ability of yeast cells within the biofilms to withstand host immune defenses. Given this clinical importance of the biofilm growth form, the aim of this study was to characterize biofilms produced by three NCAC species, namely $C$. parapsilosis, $C$. tropicalis and C. glabrata. The biofilm forming ability of clinical isolates of $C$. parapsilosis, C. tropicalis and C. glabrata recovered from different sources, was evaluated by crystal violet staining. The structure and morphological characteristics of the biofilms were also assessed by scanning electron microscopy and the biofilm matrix composition analyzed for protein and carbohydrate content. All NCAC species were able to form biofilms although these were less extensive for $C$. glabrata compared with $C$. parapsilosis and $C$. tropicalis. It was evident that $C$. parapsilosis biofilm production was highly strain dependent, a feature not evident with $C$. glabrata and C. tropicalis. Scanning electron microscopy revealed structural differences for biofilms with respect to cell morphology and spatial arrangement. Candida parapsilosis biofilm matrices had large amounts of carbohydrate with less protein. Conversely, matrices extracted from $C$. tropicalis biofilms had low amounts of carbohydrate and protein. Interestingly, C. glabrata biofilm matrix was high in both protein and carbohydrate content. The present work demonstrates that biofilm forming ability, structure and matrix composition are highly species dependent with additional strain variability occurring with $C$. parapsilosis.
\end{abstract}

Keywords Biofilm, non-Candida albicans Candida species

\section{Introduction}

Invasive fungal infections, such as candidiases, represent a public health problem of major importance [1]. Candida species normally exist as commensals but they are also opportunistic pathogens, with the ability to cause a variety of superficial and systemic infections. In the past ten

Received 4 July 2008; Received in final revised form 29 August 2008; Accepted 13 October 2008

Correspondence: Joana Azeredo, Institute for Biotechnology and Bioengineering, Universidade do Minho, Campus de Gualtar 4710057, Braga, Portugal. Tel: +351 253604419; fax: +351 253678986; E-mail: jazeredo@deb.uminho.pt years, the number of infections caused by Candida species has progressively increased [2]. This emergence is often associated with the increasing incidence of human immunodeficiency virus (HIV) infection [3], the rise in the elderly population base [4], a higher number of immunocompromised patients [5] and the more widespread use of indwelling medical devices $[6,7]$.

Although most cases of candidiases have been attributed to Candida albicans, more recently, nonCandida albicans Candida (NCAC) species (Candida parapsilosis,Candida tropicalis and Candida glabrata) have been identified as common pathogens. The prevalence of these species in human infection has been changing in

DOI: 10.3 | $09 / 13693780802549594$ 
recent years. In the 1980s, according to Kiehn et al. [8], C. albicans constituted 68\% of Candida isolates from sites other than blood in cancer patients, while C. tropicalis, C. parapsilosis and C. glabrata only accounted for 12.3 , 10.3 and $3.0 \%$ of isolates, respectively. Moreover, recently, $60 \%$ of the fungemia cases reported by Bassetti et al. [9] were due to NCAC species.

Candida tropicalis has emerged as the second or third most common agent of candidemia, mainly in oncology patients [10,11]. Moreover, the increased incidence of $C$. tropicalis as a causative agent of nosocomial urinary tract infections has been reported [12]. Candida parapsilosis is generally regarded as one of the less virulent yeast species, although it is now a frequent cause of candidemia. Nosocomial outbreaks of $C$. parapsilosis have also been described and have been attributed to transfer of the yeast from the hands of healthcare workers [13]. Candida glabrata has recently emerged as an important nosocomial pathogen, yet little is known about its epidemiology [14]. Candida glabrata is of particular importance because of its innately high resistance to certain antifungal agents, specifically the azoles [15].

One of the major contributions to Candida virulence is its versatility in adapting to a variety of different habitats and the formation of surface-attached microbial communities known as biofilms [16]. Biofilm cells are organized into structured communities embedded within a matrix of extracellular material that is produced by the biofilm cells [17]. Generally, the biofilm matrix composition includes (in addition to water), carbohydrates, proteins, phosphorus, glucose and hexosamines. However, a large portion of the biofilm matrix still remains to be identified [18]. The formation of Candida biofilms has important clinical repercussions because of their increased resistance to antifungal therapy and the protection afforded against host immune defenses $[17,19]$.

Many previous studies have focused on $C$. albicans biofilms [18,20-24] due to its well-recognized virulence, whereas only few studies of biofilms generated by all NCAC species have been reported [25-30]. Thus, the aims of this work were firstly to assess the biofilm formation ability of clinical isolates of C. glabrata, C. tropicalis and C. parapsilosis recovered from different body sites, and secondly, to characterize the biofilm structure and matrix composition in terms of protein and carbohydrate content.

\section{Materials and methods}

Organisms

A total of 18 clinical strains (Table 1) of $C$. tropicalis, $C$. glabrata and C. parapsilosis, recovered from different body sites, were used in the course of this study. The majority of isolates were recovered from vaginal and urinary tract samples and were part of the collection at the Hospital of S. Marcos, Braga, Portugal. C. tropicalis strains 12 and 75 (recovered from the vaginal tract), were obtained from the archive collection of the University of Maringá, Brazil. All oral isolates were stock isolates of the biofilm group of the Centre of Biological Engineering, and were originally isolated in the Clinic of Dentistry, Congregados, Portugal. Three reference strains of $C$. tropicalis, $C$. glabrata and C. parapsilosis from the American Type Culture Collection (ATCC) were also examined. The identity of all isolates was confirmed using CHROMagar Candida (CHROMagar, Paris, France) and by PCR-based sequencing using specific primers (ITS1 and ITS4) against the 5.8S subunit gene reference. Genomic DNA was extracted following previously described procedures [31]. The PCR products were sequenced using the ABI-PRISM Big Dye terminator cycle sequencing kit (Perkin Elmer, Applied Biosystems, Warrington, UK).

\section{Growth conditions}

For each experiment, strains were subcultured on Sabouraud dextrose agar (SDA) (Merck, Darmstadt, Germany) for $48 \mathrm{~h}$ at $37^{\circ} \mathrm{C}$. Cells were then inoculated in Sabouraud dextrose broth (SDB) (Merck) and incubated for $18 \mathrm{~h}$ at $37^{\circ} \mathrm{C}$ under agitation at $120 \mathrm{rev} / \mathrm{min}$. After incubation, the cells were harvested by centrifugation at $3000 \mathrm{~g}$ for $10 \mathrm{~min}$ at $4^{\circ} \mathrm{C}$ and washed twice with ultra-pure sterile water. Pellets were then suspended in SDB and the cellular density adjusted to $1 \times 10^{7}$ cells ml ${ }^{-1}$ using a Neubauer counting chamber.

\section{Biofilm biomass quantification}

Standardized cell suspensions (200 $\mu \mathrm{l}$ containing $1 \times 10^{7}$ cells $\mathrm{ml}^{-1}$ in SDB) were placed into selected wells of 96-well polystyrene microtiter plates (Orange Scientific, Brainel'Alleud, Belgium) and incubated at $37^{\circ} \mathrm{C}$ on a shaker at $120 \mathrm{rev} / \mathrm{min}$. At $24 \mathrm{~h}, 100 \mu \mathrm{l}$ of SDB medium was removed and an equal volume of fresh SDB added. The preparations were then incubated for a further $48 \mathrm{~h}$. After this step, the medium was aspirated and non-adherent cells removed by washing the biofilms twice with sterile ultra-pure water.

Biofilm forming ability was assessed through quantification of total biomass by crystal violet (CV) staining. Thus, after washing, biofilms were fixed with $200 \mu \mathrm{l}$ of methanol, which was removed after 15 min of contact. The microtiter plates were allowed to dry at room temperature, and $200 \mu \mathrm{l}$ of CV (1\% v/v) added to each well and incubated for $5 \mathrm{~min}$. The wells were then gently washed with sterile, ultra-pure water and $200 \mu \mathrm{l}$ of acetic acid (33\% v/v) added to release and dissolve the stain. The absorbance of the obtained solution was read in triplicate in a microtiter plate reader (Bio-Tek Synergy HT, Izasa, Lisbon, Portugal) 
Table 1 Origin, reference and biofilm matrix composition of non-Candida albicans Candida species. The values are means \pm standard deviations.

\begin{tabular}{|c|c|c|c|c|}
\hline \multirow[b]{2}{*}{ Species } & \multirow[b]{2}{*}{ Origin } & \multirow[b]{2}{*}{ Reference } & \multicolumn{2}{|c|}{ Matrix component (mg/g of biofilm dry weight) } \\
\hline & & & Protein & Carbohydrate \\
\hline \multirow[t]{7}{*}{ C. parapsilosis } & \multirow[t]{2}{*}{ Oral tract } & $\mathrm{AD}$ & $35.9 \pm 7.2$ & $748.8 \pm 43.8^{*}$ \\
\hline & & AM2 & $75.1 \pm 7.2^{*}$ & $926.8 \pm 144.9^{*}$ \\
\hline & \multirow[t]{2}{*}{ Urinary tract } & 534638 & $20.2 \pm 4.5^{*}$ & $263.7 \pm 13.2^{*}$ \\
\hline & & 553877 & $46.8 \pm 16.6$ & $592.6 \pm 93.4$ \\
\hline & \multirow[t]{2}{*}{ Vaginal } & 491861 & $80.6 \pm 16.6^{*}$ & $555.2 \pm 238.5$ \\
\hline & & 513143 & $55.3 \pm 16.6$ & $675.2 \pm 169.0$ \\
\hline & Reference & ATCC 22019 & $42.2 \pm 10.3$ & $516.4 \pm 219.1$ \\
\hline \multirow[t]{7}{*}{ C. tropicalis } & \multirow[t]{2}{*}{ Oral tract } & AG1 & $46.3 \pm 3.5$ & $22.2 \pm 5.8$ \\
\hline & & $\mathrm{T} 2.2$ & $28.2 \pm 3.3^{*}$ & $21.5 \pm 4.0$ \\
\hline & \multirow[t]{2}{*}{ Urinary tract } & 519468 & $34.2 \pm 9.3$ & $15.7 \pm 1.9$ \\
\hline & & 544123 & $41.6 \pm 1.0$ & $11.3 \pm 5.8$ \\
\hline & \multirow[t]{2}{*}{ Vaginal } & 12 & $54.0 \pm 2.1^{*}$ & $58.7 \pm 7.4^{*}$ \\
\hline & & 75 & $34.7 \pm 3.7$ & $27.5 \pm 2.8$ \\
\hline & Reference & ATCC 750 & $64.6 \pm 18.2^{*}$ & $15.5 \pm 2.8$ \\
\hline \multirow[t]{7}{*}{ C. glabrata } & \multirow[t]{2}{*}{ Oral tract } & D1 & $325.2 \pm 31.4$ & $572.8 \pm 111.2$ \\
\hline & & AE2 & $226.7 \pm 84.1$ & $241.8 \pm 52.2$ \\
\hline & \multirow[t]{2}{*}{ Urinary tract } & 562123 & $181.7 \pm 28.7$ & $409.5 \pm 112.4$ \\
\hline & & 513100 & $226.5 \pm 59.3$ & $233.7 \pm 88.5$ \\
\hline & \multirow[t]{2}{*}{ Vaginal } & 534784 & $136.4 \pm 38.5$ & $398.3 \pm 130.8$ \\
\hline & & 585626 & $246.9 \pm 47.5$ & $742.6 \pm 285.2^{*}$ \\
\hline & Reference & ATCC 2001 & $243.6 \pm 30.7$ & $420.3 \pm 39.2$ \\
\hline
\end{tabular}

*Significantly different $(P<0.05)$ for each species.

at $570 \mathrm{~nm}$. Experiments were repeated as part of three to five independent assays.

\section{Biofilm structure}

To examine the structure of biofilms by scanning electron microscopy $2 \mathrm{ml}$ of the standardized cell suspension $\left(1 \times 10^{7}\right.$ cells $\mathrm{ml}^{-1}$ in SDB) was introduced into 24-well polystyrene plates (Orange Scientific) and incubated for $48 \mathrm{~h}$ at $37^{\circ} \mathrm{C}$ and $120 \mathrm{rev} / \mathrm{min}$. After $24 \mathrm{~h}, 1 \mathrm{ml}$ of SDB medium was removed and an equal volume of fresh SDB added. At $48 \mathrm{~h}$, the medium was aspirated and non-adherent cells removed by washing the biofilms twice with sterile ultra-pure water. Samples were dehydrated with alcohol (using 70\% ethanol for $10 \mathrm{~min}, 95 \%$ ethanol for $10 \mathrm{~min}$ and $100 \%$ ethanol for $20 \mathrm{~min}$ ) and air dried for 20 min. Samples were kept in a desiccator until the base of the wells was removed for analysis. Prior to observation, the bases of the wells were mounted onto aluminum stubs, sputter coated with gold and observed with an S-360 scanning electron microscope (Leo, Cambridge, USA).

\section{Biofilm matrix composition}

Extraction method. Biofilms for analysis of matrix material were formed in 6-well polystyrene microtiter plates
(Orange Scientific). For this, inocula of $3 \mathrm{ml}$ of yeast cell suspension $\left(1 \times 10^{7}\right.$ cells $\mathrm{ml}^{-1}$ in $\left.\mathrm{SDB}\right)$ were added to each well and biofilms were formed as described previously. After $48 \mathrm{~h}$, the biofilm matrix was extracted using a slight modification to a previously described protocol [32]. Briefly, biofilm samples were scraped from the 6-well plates, resuspended with ultra-pure water, sonicated (Ultrasonic Processor, Cole-Parmer, Illinois, USA) for $30 \mathrm{~s}$ at $30 \mathrm{~W}$, and then the suspension was vortexed for $2 \mathrm{~min}$. The suspension was centrifuged at $3000 \mathrm{~g}$ for $10 \mathrm{~min}$ at $4^{\circ} \mathrm{C}$ and the supernatant filtered through a $0.2 \mu \mathrm{m}$ nitrocellulose filter and stored at $-20^{\circ} \mathrm{C}$ before analysis. The pellets were dried at $60^{\circ} \mathrm{C}$ until a constant dry biofilm weight was determined. The experiments were performed in triplicate and in three independent assays.

\section{Quantification assays}

Protein and carbohydrate quantification. The protein content of the biofilm matrix was measured using the BCA Kit (Bicinchoninic Acid, Sigma-Aldrich, St Louis, USA), using bovine serum albumin (BSA) as the standard.

Total carbohydrate content of the biofilm matrix was estimated according to the procedure of Dubois et al. [33], using glucose as the standard. 


\section{Statistical analysis}

Results were compared using One-Way analysis of variance (ANOVA) by applying Levene's test of homogeneity of variance and the Tukey multiple-comparisons test, using SPSS software (SPSS [Statistical Package for the Social Sciences], Inc., Chicago, USA). All tests were performed with a confidence level of $95 \%$.

\section{Results}

Biofilm forming ability by non-Candida albicans Candida species

Figure 1 presents the results of biofilm quantification using $\mathrm{CV}$ staining. It was evident that all NCAC species formed biofilms, although differences occurred depending on species or strain as in the case of $C$. parapsilosis. Importantly, it was noticed that generally $C$. glabrata biofilms had less total biomass (average Abs $=0.53 \pm 0.22$ ) compared with $C$. parapsilosis (average Abs $=1.14 \pm 0.43$ ) and $C$. tropicalis (average Abs $=1.31 \pm 0.08$ ).

Candida glabrata strains had similar biofilm forming ability with no significant strain differences $(P>0.05)$. In contrast, $C$. parapsilosis strains were heterogeneous in terms of the level of biofilm formation. Biofilms formed by C. parapsilosis $\mathrm{AD}$ yielded the highest level of absorbance $(\mathrm{Abs}=1.84 \pm 0.34)$ which were statistically higher than for C. parapsilosis $534638(P=0.004), 553877(P<0.001)$ and ATCC $22019(P<0.001)$. Candida tropicalis strains exhibited a more homogeneous behavior with all strains being high biofilm formers (Abs values $\geq 0.75$ ). No statistical differences were found in the extent of biofilm formation for all the $C$. tropicalis strains $(P>0.05)$, with the exception of strain 12, which had the highest biofilm forming ability $(\mathrm{Abs}=2.65 \pm 0.13)(P<0.001)$. This strain produced a two-fold greater level of biofilm than other strains of C. tropicalis and C. parapsilosis, and a five-fold increase over $C$. glabrata strains.

For each species, strains originating from the urinary tract generally yielded lower biofilm levels compared with those from other body sites. In the case of C. parapsilosis, oral isolates were the highest biofilm producers and for the other NCAC species studied, the highest biofilm producers were vaginal isolates (C. glabrata 534784 and C. tropicalis 12).

\section{Structure of non-Candida albicans Candida species biofilms}

SEM analysis was used to examine biofilm structure and to determine Candida morphological characteristics (Fig. 2).

Mature biofilms of $C$. parapsilosis and C. tropicalis strains consisted of a dense network of cells of a variety of morphologies. Biofilms of C. parapsilosis strains AD, 553877 and ATCC 22019 were composed of both yeasts and pseudohyphae, although biofilms formed by other strains of the same species were devoid of pseudohyphae (Fig. 2A). Biofilms formed by $C$. tropicalis exhibited only yeast morphology, with exception of strains AG1 and 12 which presented hyphal forms, with the latter appearing as especially long filaments (Fig. 2B). All biofilms of $C$. glabrata strains were comprised only of yeasts (Fig. 2C).

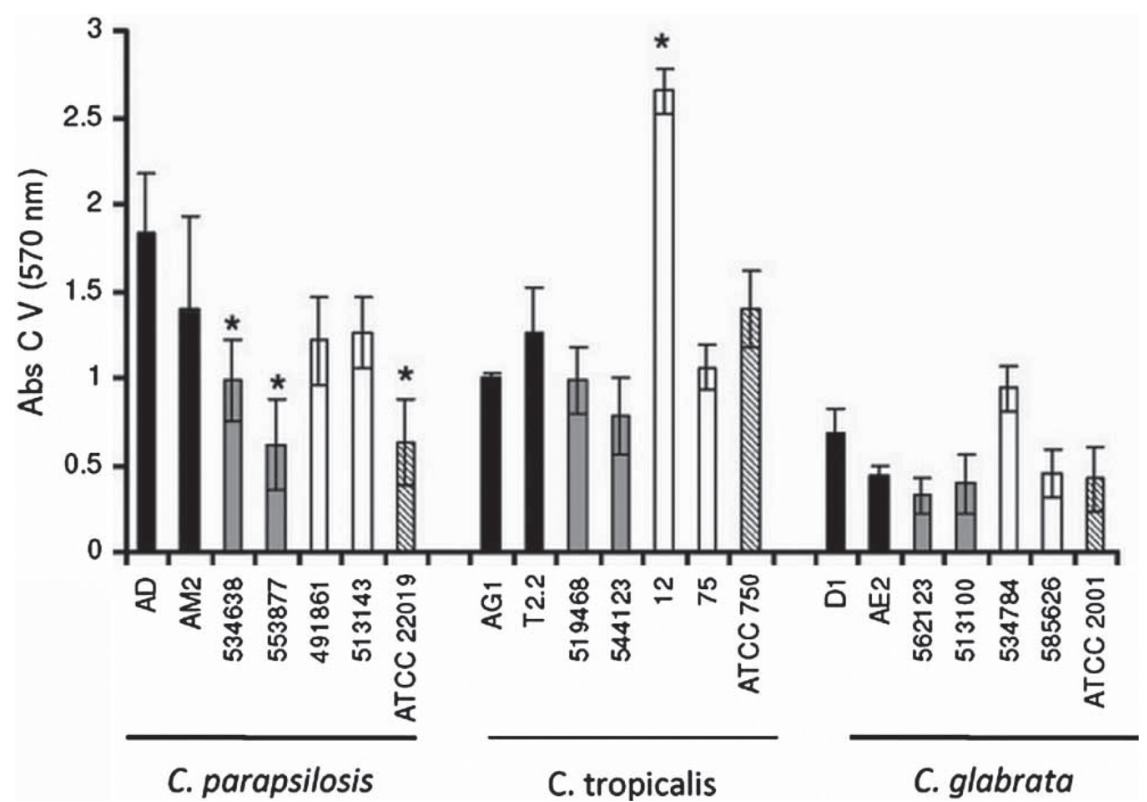

Fig. 1 Absorbance values of Crystal Violet solutions (Abs CV) obtained from $48 \mathrm{~h}$ biofilms of non-Candida albicans Candida species formed in $\operatorname{SDB}(\lambda=570 \mathrm{~nm})$ from different origins ( $\mathbf{\square}$ oral, $\square$ urinary and $\square$ vaginal). Error bars represent standard deviation. *Strains that are significantly different $(P<0.05)$ in each species. 
(A)
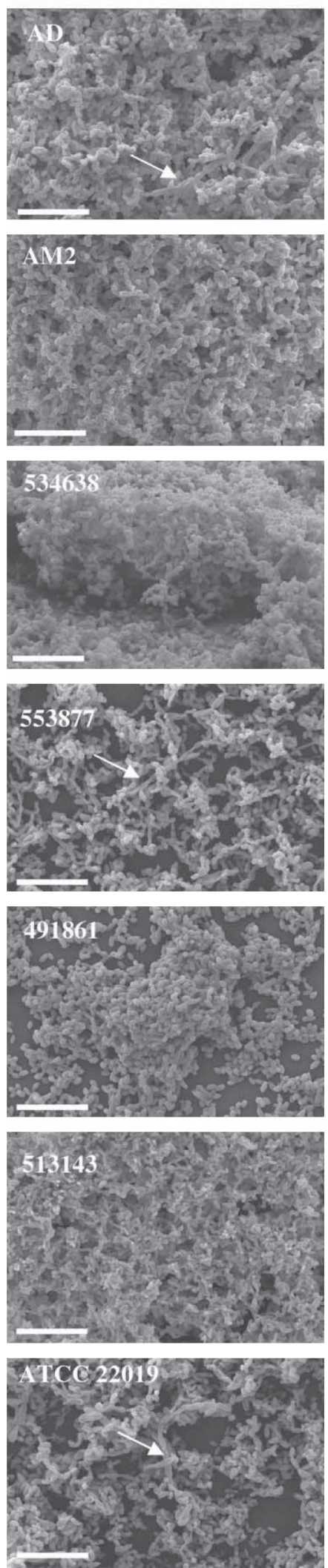

(B)
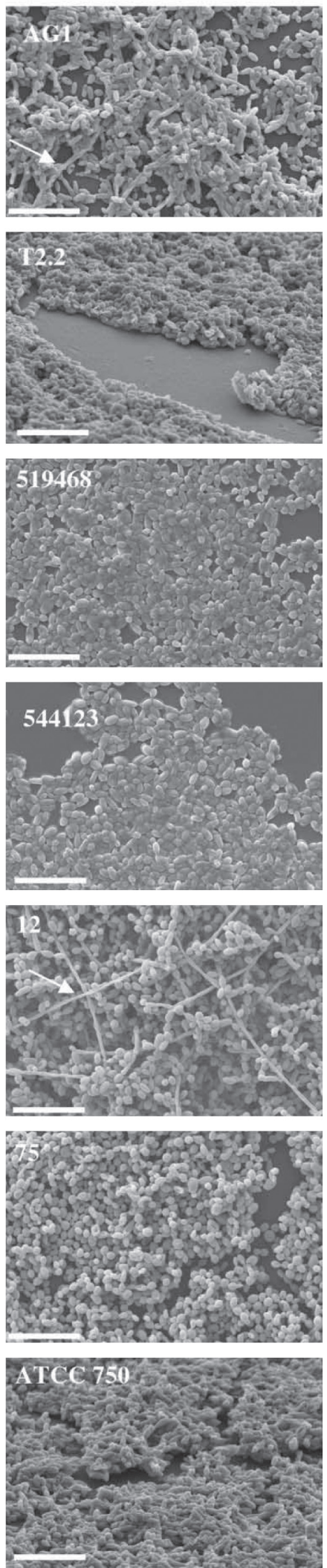

(C)
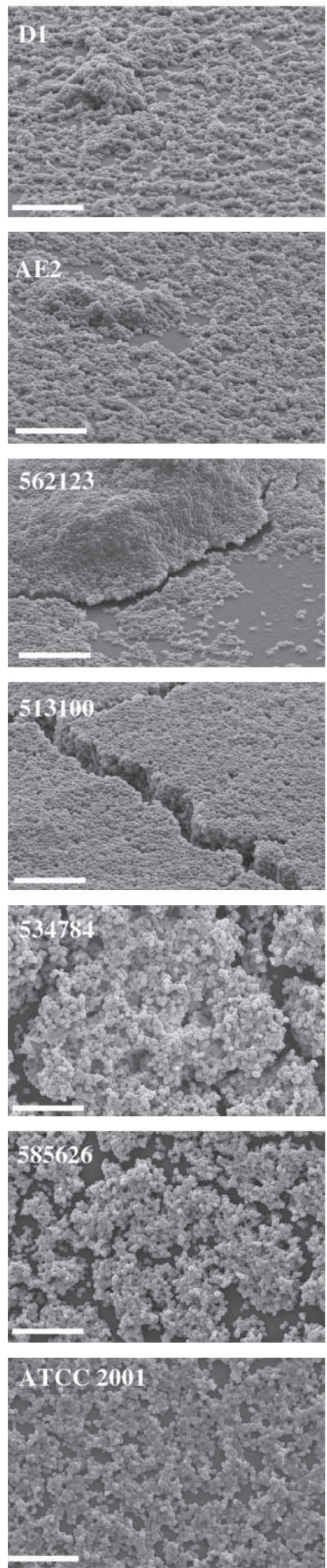

Fig. 2 (Continued)

(c) 2009 ISHAM, Medical Mycology, 47, 68I-689 
In the case of $C$. parapsilosis biofilm structure (Fig. 2A), strains AD, AM2, 534638 and 513143 formed a multilayer and compact biofilms covering the entire surface. In contrast, biofilms of strains 553877, 491861 and ATCC 22019, consisted of non-contiguous cell aggregates. The structure of $C$. tropicalis (Fig. 2B) biofilms was also strain dependent with some strains (AG1, T2.2, 12, 75 and ATCC 750) producing thick biofilms of co-aggregated cells and others (strain 519468 and 544123) yielding a more discontinuous monolayer of yeasts anchored to surface. Candida glabrata (Fig. 2C) revealed either a multilayer biofilm structure intimately packed (strains 562123 and 513100) or constituted by clusters of cells (strains D1, AE2, 534784, 585626 and ATCC 2001).

The biofilm structures for isolates from the same clinical origin were similar for C.glabrata strains (Fig. 2C). Biofilms formed by the urinary isolates (562123 and 513100) displayed a highly compact layer covering the entire surface in a 'carpet' like appearance. Biofilms of oral isolates (D1 and AE2) revealed smaller clusters compared to those in biofilms of vaginal isolates (534784 and 585626). Biofilms of oral C. parapsilosis strains (Fig. 2A) ( $\mathrm{AD}$ and $\mathrm{AM} 2$ ) were very similar, presenting a multilayer structure. In the case of C. tropicalis (Fig. 2B) urinary isolates (519468 and 544123), the biofilms were also similar, presenting as discontinuous monolayers.

\section{Matrix biofilm composition}

Table 1 shows the yield of total protein and carbohydrates extracted from biofilms formed by the NCAC species studied. The results showed that generally $C$. parapsilosis biofilm matrices had high amounts of carbohydrate (average $\mathrm{mg} / \mathrm{g}$ biofilm dry weight $=611.2 \pm 206.5$ ) and relatively lower amounts of proteins (average $\mathrm{mg} / \mathrm{g}$ biofilm dry weight $=50.9 \pm 21.4)$. Strain differences were evident in terms of both protein and carbohydrate contents, with $C$. parapsilosis AM2 having a statistically higher carbohydrate and protein content in its biofilm matrix as compared with the all other $C$. parapsilosis strains, except strain 491861 (the highest protein content) and strain $\mathrm{AD}$ (similar to AM2 in carbohydrate content).

In contrast, compared with the other species, the biofilm matrices of $C$. tropicalis strains had lower concentrations of both protein (average $\mathrm{mg} / \mathrm{g}$ biofilm dry weight $=43.4 \pm 12.7$ ) and carbohydrates (average $\mathrm{mg} / \mathrm{g}$ biofilm dryweight $=24.6 \pm 15.9$ ). Despite statistical differences being evident between $\mathrm{C}$. tropicalis strains, the relatively low protein and carbohydrate matrix composition was a consistent finding for $C$. tropicalis.

Interestingly, biofilm matrices of $C$. glabrata had relatively higher quantities of both protein (average $\mathrm{mg} / \mathrm{g}_{\text {bio- }}$ film dry weight $=226.7 \pm 58.6$ ) and carbohydrate (average $\mathrm{mg} / \mathrm{g}$ biofilm dry weight $=431.3 \pm 179.6$ ) compared with the other species. Indeed, protein levels were on average five times higher than those of C. parapsilosis and C. tropicalis.

No correlation was found concerning the amount of carbohydrate and protein extracted from biofilm of NCAC species and the respective source of each clinical isolate. However, the biofilm matrix of the vaginal clinical isolate, $C$. tropicalis 12, presented the highest amount of protein and carbohydrate of all clinical isolates of $C$. tropicalis. For $C$. parapsilosis, oral isolates (AD and AM2) had the highest quantity of carbohydrates and the urinary isolate, C. parapsilosis 534638, the lowest protein and carbohydrate content.

\section{Discussion}

Biofilm forming ability may confer NCAC species with an ecological advantage, aiding survival as commensals and pathogens of humans by allowing them to evade host immune mechanisms, resisting antifungal treatment, and withstanding the competitive pressure from other microorganisms. Biofilm formation in NCAC species, besides possibly being a key factor for the survival of these species, may also be responsible for them being particularly well adapted to colonization of tissues and indwelling devices.

In the present study, the biofilm formation ability of different clinical isolates of NCAC species was evaluated and the results (Fig. 1) showed that all NCAC species studied (C. tropicalis, C. parapsilosis and C. glabrata), formed biofilms on polystyrene surfaces under the assayed conditions, although to different extents depending on the species and strain. These results were in agreement with those of other authors, who reported that biofilm formation by Candida species occurs on a number of abiotic surfaces, including polystyrene [24-26,34]. Significant statistical differences were found for biofilm production by the NCAC species in SDB medium. In fact, C. glabrata strains were, in general, less able to form biofilms than $C$. parapsilosis and $C$. tropicalis strains. These results are in accordance with Shin et al. [26] who reported that biofilm positivity occurred most frequently in isolates of C. tropicalis, followed by C. parapsilosis and C. glabrata.

Fig. 2 Scanning electron microscopy of non-Candida albicans Candida species biofilms formed in SDB at 48 h. (A) C. parapsilosis, (B) C. tropicalis and (C) C. glabrata clinical isolate strains. Arrows indicate the presence of hyphal morphologies. The bar in the images corresponds to $20 \mu \mathrm{m}$. Magnification $\times 1000$. 
It was noted that the biofilm forming ability of C. parapsilosis species was highly strain dependent, which was less evident with both $C$. glabrata and C. tropicalis. These observations corroborate previous reports for C. albicans whose growth and virulence attributes, together with biofilm formation [23,25] have been shown to be highly strain dependent. Such findings undoubtedly reflect inherent physiological differences between strains and could have significance with respect to pathogenic potential.

Despite the inherently destructive nature of SEM processing, the method provided useful information on biofilm structure and on the different cellular morphologies. It is known that biofilm structure is dependent on environmental factors including growth conditions, nature of colonized surface $[22,25,29]$ and importantly from the perspective of this present study, the microbial species and strains involved $[22,25,29,34,35]$. SEM did indeed reveal structural and morphological differences for the biofilms of the studied NCAC species and strains. Biofilms of $C$. glabrata (Fig. 2C) presented as a multilayered structure with blastoconidia intimately packed, for some strains, and for others as a biofilm composed of cell clusters. As expected, there was a total absence of pseudohyphae and hyphae since C. glabrata is a non-hyphal species. Recently, Zaw et al. [36] also reported that after $48 \mathrm{~h}$, the biofilms of aerobically grown $C$. glabrata generally revealed a multilayer structure packed with blastoconidia devoid of pseudohyphae and hyphae. In the presented study, C. parapsilosis strains (Fig. 2A) yielded a multilayer biofilm structure that was comprised of a dense network of yeasts and pseudohyphae. Although few studies on the biofilm structure of $C$. parapsilosis strains have been reported, Kuhn et al. [35] described that C.parapsilosis biofilms consisted of irregular groupings of blastoconidia on a basal blastoconidia layer. Regarding C. tropicalis, its biofilms appeared as discontinuous layers of large blastoconidia anchored to the surface, which was in accordance with the findings of Bizerra et al. [30]. The latter also reported that $C$. tropicalis biofilms formed in SDB medium, contained only blastoconidia or generated a multilayer heterogeneous structure covering the entire surface as a thick biofilm. In the present study, large quantities of hyphal elements were found in strain C. tropicalis 12 biofilms (vaginal clinical isolate). It has been suggested $[21,37]$ that the presence of such hyphae may have importance in the structural integrity of multilayered biofilms. The present study reinforces and emphasizes a previous study where one $C$. tropicalis strain formed a thin layer of hyphae (in YNB) compared with other strains only presenting blastoconidia [35]. Parahitiyawa et al. [34], reported that on polystyrene surfaces, C. tropicalis biofilms consisted of large coaggregated microcolonies of blastoconidia with a thick extracellular polymeric layer. In fact, almost all microorganisms display structural heterogeneity within their biofilm architecture [38]. The present work indicates that this heterogeneity appears to be common in biofilms formed by $C$. glabrata, $C$. tropicalis and $C$. parapsilosis strains, revealing new important aspects on NCAC species biofilm ultrastructure.

One of the most important characteristics of both bacterial and fungal biofilms is the presence and composition of the extracellular matrix $[17,28]$. There is a general consensus that the biofilm matrix acts as a barrier to diffusion of antimicrobial agents, thereby limiting access of antimicrobials to organisms at the base of the biofilm [39]. In this study, biofilm matrices were analyzed for carbohydrate and protein content. Significantly, consistent differences were found in the composition of biofilms of the NCAC species. Matrices isolated from C. parapsilosis biofilms consisted of high amounts of carbohydrates and small amounts of proteins, whilst $C$. tropicalis biofilms were low in both carbohydrate and protein content. These results are in accordance with previous work [28] on C. tropicalis biofilm matrices which that they were mainly composed of hexosamine, with smaller amounts of carbohydrate and proteins. To the authors' knowledge, this is the first report on the analysis of the biofilm matrices of $C$. parapsilosis and C.glabrata. Interestingly, the matrices recovered from C. glabrata strains had higher amounts of both proteins and carbohydrates. This is an interesting result, especially when related to potential virulence of this species whose infections yield both the highest mortality rate [40] and resistance to antifungal agents [15].

The three different sources (body sites) for the clinical isolates represent very diverse ecological niches and differ in many biotic and abiotic factors. Recent reports have demonstrated that blood isolates produce greater quantities of biofilm compared with oral isolates [41]. In this current study, no correlation was found in terms of biofilm forming ability and matrix composition with the origin of the isolate. However, biofilm structure analysis did highlight some interesting aspects. For $C$. glabrata, the biofilm structure for isolates from the same origin did appear to be similar. This was also true for $C$. tropicalis urinary tract isolates. It could readily be hypothesized that for certain body sites, colonization requires a particular phenotype with respect to biofilm formation. Such a biofilm phenotype might be genetically rather than environmentally governed, thus explaining why the biofilm structural differences could be detected in these in vitro studies. Through elucidating such inherent differences, it might be possible to identify and specifically combat strains adapted for infection at particular body sites. It must be emphasized, however, that further investigations with isolates from specific environments are required to confirm this hypothesis. 


\section{Acknowledgements}

The authors acknowledge 'Fundação para a Ciência e Tecnologia (FCT)', Portugal, for supporting Sonia Silva work through grant SFRH/BD/28341/2006 and project PDTC/BIO/61112/2004.

Declaration of interest: The authors report no conflicts of interest. The authors alone are responsible for the content and writing of the paper.

\section{References}

1 Fridkin SK, Jarvis WR. Epidemiology of nosocomial fungal infections. Clin Microbiol Rev 1996; 9: 449-511.

2 Odds FC. Candida and Candidosis, 2nd edn. London: Bailliere Tindall, 1998.

3 Samaranayake LP, Fidel PL, Naglik JR, et al. Fungal infections associated with HIV infection. Oral Dis 2002; 8: 151-160.

4 Fanello S, Bouchara JP, Jousset N, Delbos V, LeFlohic AM. Nosocomial Candida albicans acquisition in a geriatric unit: epidemiology and evidence for person-to-person transmission. J Hosp Infect 2001; 47: 46-52.

5 Hargety JA, Ortiz J, Reich D, Manzarbeitia C. Fungal infections in solid organ transplant patients. Surg Infect (Larchmt). 2003; 4: 263-271.

6 Kojic EM, Darouiche RO. Candida infections of medical devices. Clin Microbiol Rev 2004; 17: 255-267.

7 Kumamoto CA. Candida biofilms. Curr Opin Microbiol 2002; 5: 608-611.

8 Kiehn TE, Edwards FF, Armstrong D. The prevalence of yeasts in clinical specimens from cancer patients. Amer J Clin Pathol 1980; 73: 518-521.

9 Bassetti M, Righi E, Costa A, et al. Epidemiological trends in nosocomial candidemia in intensive care. BMC Infect Dis 2006; 6: 21.

10 Weinberger M, Leibovici L, Perez S, et al. Characteristics of candidaemia with Candida albicans compared with non albicans Candida species and predictors of mortality. J Hosp Infect 2005; 61: 146-154.

11 Nucci M, Colombo AL. Candidemia due to Candida tropicalis: clinical, epidemiologic and microbiologic characteristics of 188 episodes occurring in tertiary care hospitals. Diagn Microbiol Infect Dis 2007; 58: $77-82$.

12 Rho J, Shin JH, Song JW, et al. Molecular investigation of two consecutive nosocomial clusters of Candida tropicalis candiduria using pulsed-field gel electrophoresis. J Microbiol 2004; 42: 80-86.

13 Bonassoli LA, Bertoli M, Svidzinski TIE. High frequency of Candida parapsilosis on the hands of healthy hosts. J Hosp Infect 2005; 59: 159-162.

14 Hajjeh RA, Sofair AN, Harrison LH, et al. Incidence of bloodstream infections due to Candida species and in vitro susceptibilities of isolates collected from 1998 to 2000 in a population-based active surveillance program. J Clin Microbiol 2004; 42: 1519-1527.

15 Tsai H, Bobek LA. Studies of the mechanism of human salivary histatin-5 candidacidal activity with histatin-5 variants and azolesensitive and -resistant Candida species. Antimicrob Agents Chemother 1997; 41: 2224-2228.

16 Costerton JW, Lewandowski Z, Caldwell DE, Korber DR, Lappin-Scott HM. Microbial biofilms. Аппи Rev Microbial 1995; 49: 711-745.

17 Donlan RM, Costerton JW. Biofilms: survival mechanisms of clinically relevant microorganisms. Clin Microbiol Rev 2002; 15: 167-193.
18 Baillie GS, Douglas LJ. Matrix polymers of Candida biofilms and their possible role in biofilm resistance to antifungal agents. J Antimicrob Chemother 2000; 46: 397-403.

19 Mukherjee PK, Chandra J. Candida biofilm resistance. Drug Resist Update 2004; 7: 301-309.

20 Hawser SP, Douglas LJ. Resistance of Candida albicans biofilms to antifungal agents in vitro. Antimcrob Agents Chemother 1995; 39: 2128-2131.

21 Baillie GS, Douglas LJ. Role of dimorphism in the development of Candida albicans biofilm. J Med Microbiol 1999; 48: 671-679.

22 Chandra J, Kuhn DM, Mukherjee PK, et al. Biofilm formation by the fungal pathogen Candida albicans: development, architecture and drug resistance. J Bacteriol 2001; 183: 5385-5394.

23 Jin Y, Yip KH, Samaranayake YH, Yau JY, Samaranayake LP. Biofilm-forming ability of Candida albicans is unlikely to contribute to high levels of oral yeast carriage in cases of human immunodeficiency virus infection. J Clin Microbiol 2003; 41: 2961-2967.

24 Ramage G, Martinez JP, Lopez-Ribot JL. Candida biofilms on implanted biomaterials: a clinically significant problem. FEMS Yeast Res 2006; 6: 979-986.

25 Hawser SP, Douglas LJ. Biofilm formation by Candida species on the surface of catheter materials in vitro. Infect Immun 1994; 62: 915-921.

26 Shin JH, Kee SJ, Shin MG, et al. Biofilm production by isolates of Candida species recovered from nonneutropenic patients: comparison of bloodstream isolates with isolates from other sources. $J$ Clin Microbiol 2002; 40: 1244-1248.

27 Samaranayake YH, Ye J, Yau JYY, Cheung BPK, Samaranayake LP. In vitro method to study antifungal perfusion in Candida biofilms. J Clin Microbiol 2005; 43: 818-825.

28 Al-Fattani MA, Douglas LJ. Biofilm matrix of Candida albicans and Candida tropicalis: chemical composition and role in drug resistance. J Med Microbiol 2006; 55: 999-1008.

29 Jain N, Kohli R, Cook E, et al. Biofilm formation by and antifungal susceptibility of Candida isolates from urine. Appl Environ Microbiol 2007; 73: 1693-1703.

30 Bizerra CF, Nakamura CV, Poersch C, et al. Characteristics of biofilm formation by Candida tropicalis and antifungal resistance. FEMS Yeast Res 2008; 8: 442-450.

31 Scherer S, Stevens DA. Application of DNA typing methods to epidemiology and taxonomy of Candida species. J Clin Microbiol 1987; 25: 675-679.

32 Azeredo J, Henriques M, Sillankorva S, Oliveira R. Extraction of exopolymers from biofilms: the protective effect of glutaraldehyde. Water Sci Technol 2003; 47: 175-179.

33 Dubois M, Gilles KA, Hamilton JK, Rebers PA, Smith F. Colorimetric method for determination of sugars and related substances. Anal Chem 1956; 28: 350-356.

34 Parahitiyawa NB, Samaranayake YH, Samaranayake LP, et al. Interspecies variation in Candida biofilm formation studied using the Calgary biofilm device. APMIS 2006; 114: 298-306.

35 Kuhn DM, Chandra J, Mukherjee PK, Ghannoum MA. Comparison of biofilms formed by Candida albicans and Candida parapsilosis on bioprosthetic surfaces. Infect Immun 2002; 70: 878-888.

36 Zaw MT, Samaranayake YH, Samaranayake LP. In vitro biofilm formation of Candida albicans and non-albicans Candida species under dynamic and anaerobic conditions. Arch Oral Biol 2007; 52: 761-767.

37 Ramage G, VandeWalle K, Bachmann SP, Wickes BL, Lopez-Ribot JL. In vitro pharmacodynamic properties of three antifungal agents against preformed Candida albicans biofilms determined by time-kill studies. Antimicrob Agents Chemother 2002; 46: 3634-3636. 
38 Wimpenny J, Manz W, Szewzyk U. Heterogeneity in biofilms. FEMS Microbiol Rev 2000; 24: 661-671.

39 Al-Fattani MA, Douglas LJ. Penetration of Candida biofilms by antifungal agents. Antimicrob Agents Chemother. 2004; 48: 3291-3297.

This paper was first published online on iFirst on 20 March 2009.
40 Kcrmery K Jr. Torulopsis glabrata an emerging pathogen in cancer patients. Int J Antimicrob Agents 1999; 11: 1-6.

41 Kumar CPG, Menon T. Biofilm production by clinical isolates of Candida species. Med Mycol 2006; 44: 99-101. 